\title{
Aceratherium incisivum (RHINOCEROTIDAE) EN EL MIOCENO SUPERIOR DE CERRO DE LOS BATALLONES (MADRID)
}

\author{
Esperanza CERDEÑO $O^{\prime .2}$ y Begoña SÁNCHEZ' \\ ' Dpto. Paleobiología. Museo Nacional CC. Naturales (CSIC). José Gutiérrez \\ Abascal, 2. 28006 Madrid, España. \\ ? IANIGLA-CRICYT. Parque Gral. San Martín. C.C. 330. 5500 Mendoza, \\ Argentina.
}

Cerdeño, E. y Sánchez, B. 1998. Aceratherium incisivum (Rhinocerotidae) en el Mioceno superior de Cerro de los Batallones (Madrid). [Aceratherium incisivum (Rhinocerotidae) from the late Miocene of Cerro de los Batallones (Madrid)]. Revista Española de Paleontología, 13 (1), 51-60. ISSN 0213-6937.

\begin{abstract}
The rhinocerotid remains from Cerro de los Batallones (Madrid, Spain) are described in this paper. Their preliminary ascription to Aceratherium incisivum Kaup is confirmed. Most of the remains belong to an individual, and the comparative study shows that it has the mandibular symphysys relatively longer and the height of the vertical ramus somewhat shorter than other populations of the species; in addition, the scaphoid presents three lateral facets for the semilunar, what is uncommon in Aceratherium and closer genera. Dimensions are in general lesser than those of the type material from Eppelsheim (Germany), but they fall into the range established for the species from different European localities. Cerro de los Batallones is considered to be late Vallesian (late Miocene) in age. A. incisivum is well represented in Europe during the Vallesian and Turolian, although it has been scarcely recorded in Spain, except in the Vallés-Penedés basin. This new material enlarges the knowledge of this species in the central basins of Spain.
\end{abstract}

Keywords: Aceratherium, Rhinocerotidae, Vallesian, late Miocene, Spain.

\section{RESUMEN}

Se describen los restos de rinocerótido procedentes del yacimiento de Cerro de los Batallones (Madrid, España). Se confirma su identificación preliminar como Aceratherium incisivum Kaup. La mayor parte de los restos pertenece al mismo individuo y su estudio comparativo muestra que tiene la sínfisis mandibular relativamente más larga y la altura de la rama ascendente algo más corta que otras poblaciones de la especie; además, el escafoides presenta tres facetas de articulación con el semilunar, lo cual no es común en Aceratherium y géneros afines. En general, las dimensiones de los restos son menores que las del material tipo de Eppelsheim (Alemania), pero entran en el rango de variación establecido para la especie en diversas localidades europeas. Cerro de los Batallones se considera de edad Vallesiense superior (Mioceno superior). A. incisivum se conoce bien a lo largo del Vallesiense y Turoliense en Europa, pero está poco representado en España, salvo en la cuenca del Vallés-Penedés. Este nuevo material aumenta el conocimiento de la especie en las cuencas centrales de España.

Palabras clave: Aceratherium, Rhinocerotidae, Vallesiense, Mioceno superior, España.

\section{INTRODUCCIÓN}

El yacimiento de Cerro de los Batallones se descubrió en 1991 con motivo de unos trabajos de prospección de sepiolita por parte de la empresa TOLSA. Se encuentra situado en el término municipal de Torrejón de Velasco y constituye uno de los yacimientos más importantes del área de Madrid (Morales et cl., 1992). La característica más sorprendente de este yacimiento es la gran cantidad de restos de carnívoros que ha proporcionado, incluyendo cráneos y esqueletos casi completos de nueve especies diferentes de félidos, anficiónidos, hiénidos y mustélidos. Aparte de ellos, entre los macromamíferos, se han encontrado escasos restos de mastodonte, Hipparion, un suido indeterminado, Hispanomeryx, un cérvido y un rinoceronte que se identificó preliminarmente con Aceratherium incisivum. Además, se han recogido restos de diversas especies de insectívoros, roedores y lagomorfos, así como de tortugas y aves indeterminadas. El conjunto de la fauna recuperada es propio del 


\begin{tabular}{||l|rr|r|rr||}
\hline \hline Dimensiones mandibulares & \multicolumn{1}{|c|}{ CBAT } & CLLO & \multicolumn{2}{|c||}{ EPPE } \\
\hline \hline I sínfisis-talón & 479,5 & 479,0 & 495,0 & 502,0 & 525,0 \\
L sínf. post.-talón & 342,5 & 343,0 & 415,0 & & \\
L rama ascendente (nivel oclusal) & 128,0 & 129,0 & & \\
DT cóndilo & 92,3 & 92,0 & 95,5 & 93,3 & 95,5 \\
DT máximo sínfisis & $(94)$ & & & 108,0 & 119,0 \\
DT sínfisis, entre diastemas & 56,0 & & & \\
L sínfisis & 142,9 & & 99,0 & 130,0 & 127,6 \\
DT máximo a nivel de los I2 & 83,5 & & & \\
DT mínimo entre los I2 & 39,0 & & \\
H rama ascendente en la apófisis & 251,0 & 241,0 & 294,0 & & \\
H rama ascendente en el cóndilo & 177,0 & 162,0 & 235,0 & 251,0 & 250,0 \\
H rama horizontal P3-P4 & $(52,5)$ & & 75,0 & & \\
H rama horizontal P4-M1 & $(63,5)$ & 77,0 & 77,4 & 77,0 \\
DT rama horizontal P3-P4 & $(49,0)$ & & 42,0 & 44,1 \\
DT rama horizontal P4-M1 & $(45,0)$ & & & \\
\hline
\end{tabular}

Tabla 1. Dimensiones mandibulares de A. incisivum del Cerro de los Batallones. Se incluyen las dimensiones de los ejemplares de Can Llobateres (Santafé, 1978) y Eppelsheim (moldes del material tipo).

Vallesiense superior, dentro del Mioceno superior (Morales et al., 1992).

El material correspondiente al rinoceronte se describe en este trabajo y se confirma su atribución a la especie Aceratherium incisivum Kaup, 1834, bien conocida a lo largo del Vallesiense y Turoliense de Europa occidental (Guérin, 1980). Su representación es escasa en España. Santafé (1978) describió el material más abundante, que corresponde a localidades de la cuenca del VallésPenedés (parte descrito como A. bi-tetradactylumincisivum, considerado por Guérin, 1980 y Cerdeño, 1989, como A. incisivum); Santafé y Casanovas (1982, 1992) añaden un resto procedente de Piera y otros de Polinyá. Otros restos aislados asignados a esta especie proceden de los yacimientos de Los Valles de Fuentidueña (Segovia) y La Roma 2, Masía del Barbo, Los Mansuetos y Concud (Teruel), correspondientes al Vallesiense y Turoliense inferior y medio (Alberdi et al., 1981; Santafé y Casanovas, 1984; Cerdeño, 1989, 1992).

Clásicamente y hasta hace relativamente poco tiempo, se incluyeron en el género Aceratherium numerosas especies desde el Oligoceno al Mioceno superior, tanto de Europa como de otros continentes. Al aumentar el conocimiento de muchas de ellas, se fueron estableciendo nuevos géneros, aunque la sistemática del grupo no está totalmente aclarada. Una de las especies que más se ha relacionado con $A$. incisivum (especie tipo del género Aceratherium) es Acerorhinus tetradactylum (Lartet, 1851). Generalmente incluida en Aceratherium, Ginsburg y Heissig (1989) reconsideran su atribución y crean el género Hoploaceratherium para esta especie, añadiendo la posibilidad de que la especie asiática Acerorhinus depereti (Borissiak, 1927) pueda pertenecer también a este género. Sin embargo, la revisión reciente de unos restos de aceraterino de Tung-gur (Mongolia interior, China) (Cerdeño, 1996) lleva a la conclusión de que $A$. tetradactylum no se distingue a nivel genérico de las formas euroasiáticas referidas a Acerorhinus Kretzoi, 1942. Todas estas especies se diferencian bien, especialmente a nivel craneal, de la especie Aceratherium incisivum, si bien no dejan de ser formas estrechamente relacionadas (Cerdeño, 1995). Por otra parte, es necesario revisar otras especies atribuidas a este género, como algunas del Mioceno de Africa, para establecer definitivamente sus afinidades genéricas.

\section{MATERIAL Y METODOLOGÍA}

Los restos estudiados de rinocerótido son los siguientes: B5261-2, fragmento de $\mathrm{D}^{(4)}$ y fragmento de $\mathrm{D}^{(33)} ; \mathrm{B} 2788$, mandíbula completa, con la serie $\mathrm{P}_{3}-\mathrm{M}_{3}$ derecha y $\mathrm{P}_{2}-\mathrm{M}_{3}$ izquierda; $\mathrm{B} 461$, un $\mathrm{P}^{2}$ derecho aislado, sin desgaste, no correspondiente a la mandíbula; B791, bloque incluyendo la escápula, el fémur y parte de los huesos del tarso y metatarso izquierdos, estos últimos en conexión: B791-7, navicular; B791-5, cuboides; B791-6, entocuneiforme; B791-4, ectocuneiforme; B791-1, MtII; B791-8, MtIV; B791-2 y 3, primera y segunda falanges del MtII; y B791-9, sesamoideo; B598-1, húmero izquierdo; B1530, escafoides izquierdo; B221, fémur derecho; B303, rótula derecha; B186, tibia derecha; B2622, MtIII derecho; B581, falange primera central; B4887, falange segunda central; sin sigla: $\mathrm{P}_{\text {}}$ derecho; escafoides y pisiforme derechos.

\section{Lámina I}

Aceratherium incisivum Kaup del Cerro de los Batallones

1 Mandíbula. B2788. Vista lateral.

2 Mandíbula. B2788. Serie inferior izquierda.

3 P2 inferior derecho. B461. a: vista labial; b: vista lingual.

4 Pisiforme derecho. s/s. Vista lateral.

5 Escafoides derecho. s/s. a: vista proximal; b: vista lateral.

6 Rótula derecha. B303. Vista posterior. 


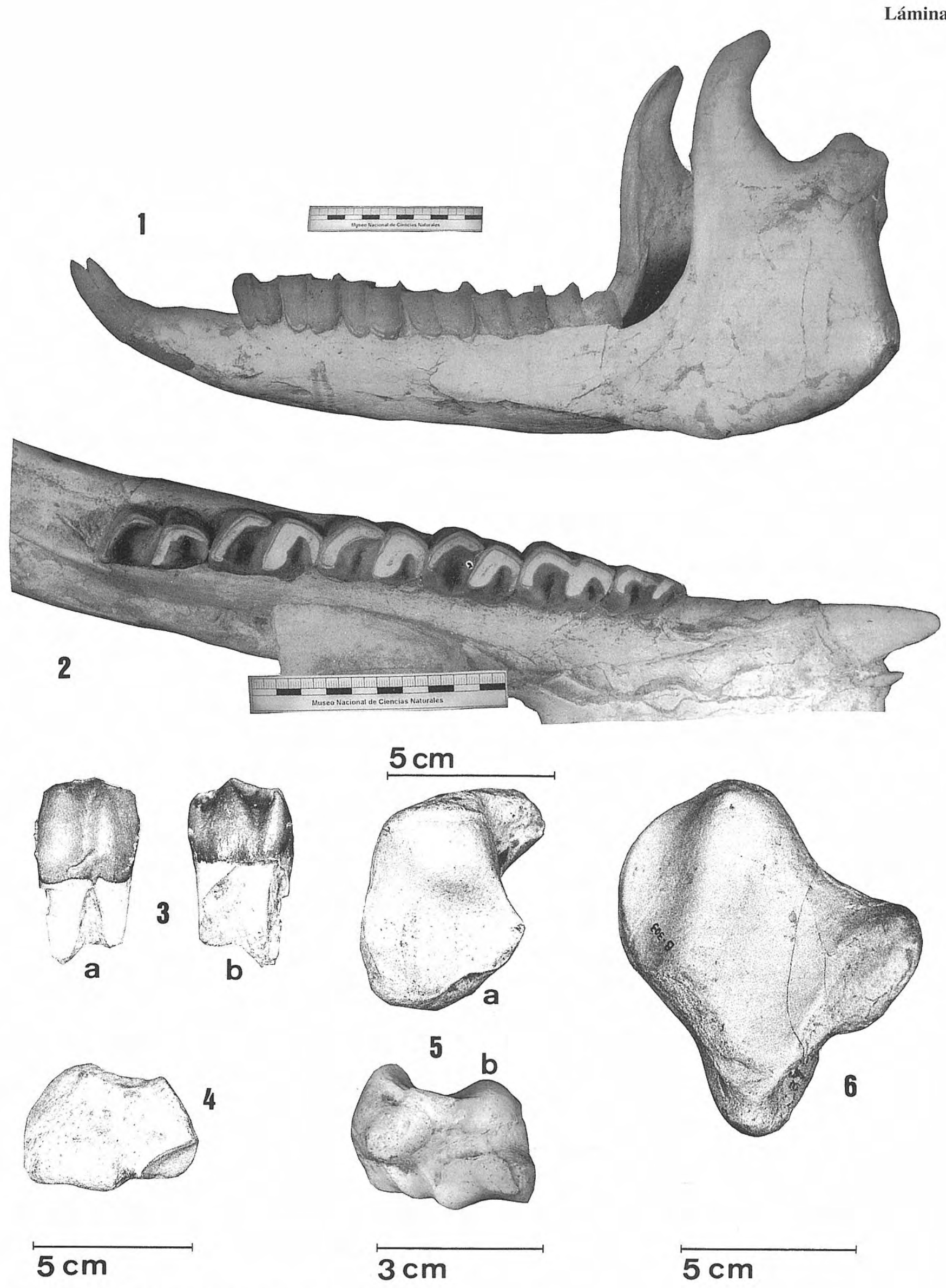

Revista Española de Paleontología, 13 (1), 1998. 


\begin{tabular}{||l|cc|l|rlr||}
\hline \multicolumn{6}{|c||}{ Dentición inferior } \\
\hline \hline & \multicolumn{2}{|c|}{ CBAT } & CLLO & \multicolumn{3}{l|}{ EPPE } \\
\hline L.P & 100 & 103 & 110 & 90,4 & 100,4 & 97,9 \\
L.M & 128 & 129 & 134 & 113,1 & 126,1 & 128,5 \\
L.P-M & $(227)$ & 232 & 243,5 & & & \\
\hline
\end{tabular}

\begin{tabular}{||c|cc|cc|cc||}
\hline & \multicolumn{2}{|c|}{ CBAT } & CLLO & \multicolumn{2}{c||}{ EPPE } \\
\hline \hline & L & A & & & L & A \\
\hline I1 & 4,7 & 4,7 & & & & \\
\hline P1 & 11,8 & 10,9 & & & 15,9 & 14,5 \\
& & & & & 15,6 & 13,2 \\
\hline \multirow{3}{*}{ P2 } & 30,0 & 19,7 & 31,5 & 20,0 & 27,8 & 22,6 \\
& 29,2 & 19,7 & 30,9 & 20,4 & 25,9 & 22,2 \\
& & & & & 26,4 & 20,1 \\
\hline \multirow{3}{*}{ P3 } & 36,0 & 26,1 & 38,4 & 27,7 & 35,2 & 26,5 \\
& 35,8 & 26,7 & 39,3 & 27,3 & 33,7 & 27,6 \\
& & & & & 31,4 & 27,1 \\
\hline \multirow{3}{*}{ P4 } & 35,0 & 27,1 & 39,0 & 28,0 & 37,3 & 30,0 \\
& 36,8 & 28,0 & 38,8 & 26,4 & 38,3 & 30,1 \\
& & & & & 32,4 & 29,3 \\
\hline \multirow{3}{*}{ M1 } & 40,8 & 26,9 & 44,1 & 31,5 & 43,1 & 30,5 \\
& 41,0 & 26,0 & 43,6 & 29,1 & 39,3 & 28,8 \\
& & & & & 34,5 & 27,1 \\
\hline \multirow{2}{*}{ M2 } & 41,0 & 27,5 & 45,0 & 28,4 & 41,7 & 33,3 \\
& 41,6 & 27,4 & 46,2 & 27,5 & 49,0 & 30,6 \\
& & & & & 39,8 & 28,7 \\
\hline \multirow{3}{*}{ M3 } & 41,4 & 25,7 & 43,2 & 26,8 & 41,2 & 29,6 \\
& 41,5 & 26,0 & 42,5 & 26,5 & 40,1 & 29,3 \\
& & & & & 38,7 & 27,1 \\
\hline \hline
\end{tabular}

Tabla 2. Dimensiones de la dentición de A. incisivum del Cerro de los Batallones. Se incluyen las dimensiones de los ejemplares de Can Llobateres (Santafé, 1978) y Eppelsheim (moldes del material tipo).

Todos ellos han sido restaurados en el Museo Nacional de Ciencias Naturales de Madrid y forman parte de su colección de vertebrados fósiles.

La mayoría de los restos parece corresponder a un mismo individuo $\mathrm{y}$, dado que muchos han aparecido articulados o asociados en un área concreta (Cerdeño, 1993, foto 3), es posible que el resto del esqueleto aparezca en futuras excavaciones, ya que el área excavada del yacimiento es aún relativamente pequeña (Morales et al., 1993). El hallazgo de dos dientes de leche superiores muy gastados y de un $\mathrm{P}_{2}$ derecho distinto al que correspondería a la mandíbula, implica un número mínimo de dos (quizá tres) individuos, uno subadulto (el individuo mejor representado) y uno (quizá dos) aún joven (los dientes de leche muy gastados y el $\mathrm{P}_{2}$ sin desgaste podrían corresponder al mismo individuo).
Este estudio comparativo sigue la metodología utilizada en trabajos previos de rinocerótidos, como los de Guérin (1980) y Cerdeño (1989). Hay que señalar que para algunos huesos, como los metápodos, debido a la torsión de la epífisis respecto a la diáfisis, el criterio de Cerdeño (op. cit.) hace que los diámetros transversal y antero-posterior de la diáfisis sean contrarios a los considerados por otros autores, y de ahí, la diferencia en las dimensiones de la diáfisis del MtII (tabla 5) con respecto a los valores de Guérin (1980) o Santafé (1978), que en realidad no son tales diferencias.

Todas las medidas se expresan en milímetros.

Abreviaturas utilizadas: $\mathrm{A}=$ anchura; ant.= anterior; apóf. $=$ apófisis; art. $=$ articulación $;$ CBAT $=$ Cerro de los Batallones; CLLO = Can Llobateres; cr.d.= cresta deltoidea (húmero); DAP= diámetro antero-posterior; d./dis.=distal; $\mathrm{DT}=$ diámetro transversal; ep.= epífisis; $\mathrm{EPPE}=$ Eppelsheim; H= altura; L= longitud; m./máx.= máximo; mín. $=$ mínimo; post $=$ posterior $;$ prox.$=$ proximal $; 3$ tr.$=$ tercer trocánter (fémur).

\section{DESCRIPCIÓN}

La mandíbula (Lám. I, figs. 1-2; Tab. 1) está muy bien conservada aunque algo deformada; mantiene las dos series yugales (salvo el $\mathrm{P}_{2}$ derecho; no hay $\mathrm{P}_{1}$ ), los dos $\mathrm{I}_{2}$ y el $\mathrm{I}_{1}$ izquierdo. Perteneció a un individuo joven-subadulto, ya que los dientes yugales presentan poco desgaste y los $I_{2}$ no han acabado de salir por completo (no se han tomado sus dimensiones).

La rama horizontal es larga y no muy alta. La rama ascendente tiene el borde posterior ancho; el cóndilo es ancho y robusto y la apófisis coronoides es alta y curvada hacia dentro y hacia atrás. La sínfisis mandibular es ancha y su límite posterior alcanza el nivel de la parte posterior de $\mathrm{P}_{3}$. Los $\mathbf{I}_{2}$ están dirigidos hacia delante, suavemente curvados y de sección triangular.

Los dientes yugales inferiores (Tab. 2; Lám. I, figs. 12) son grandes, con el surco externo bien marcado. En los $P_{3}$, existe un doble surco labial que limita una pequeña zona más aplanada entre los dos lóbulos del diente. Los premolares tienen la cara lingual algo abombada, presentando un aspecto más rechoncho que los molares. El cíngulo anterior se prolonga lingualmente, rasgo que Guérin (1980: 242) señala como característico del género Aceratherium. Labialmente, hay cíngulo más o menos continuo en los premolares y en la base del surco en los molares. El valle posterior es más profundo que el anterior.

Los dos fragmentos de dientes superiores corresponden a dientes de leche y tienen un grado de desgaste máximo.

Esqueleto postcraneal (Tabs. 3-5).

La escápula está casi completa (Lám. II, fig. 3). Es un hueso alto y relativamente estrecho (Tab. 3); el arranque de

\section{Lámina II}

Aceratherium incisivum Kaup del Cerro de los Batallones

1 Mt III derecho. B2622. Vista anterior

2 Tibia derecha. B186. Vista anterior.

3 Escápula izquierda. B791. Vista lateral
4 Fémur derecho. B221. Vista anterior.

5 Húmero izquierdo. B-598-1. Vista anterior. 
Lámina II

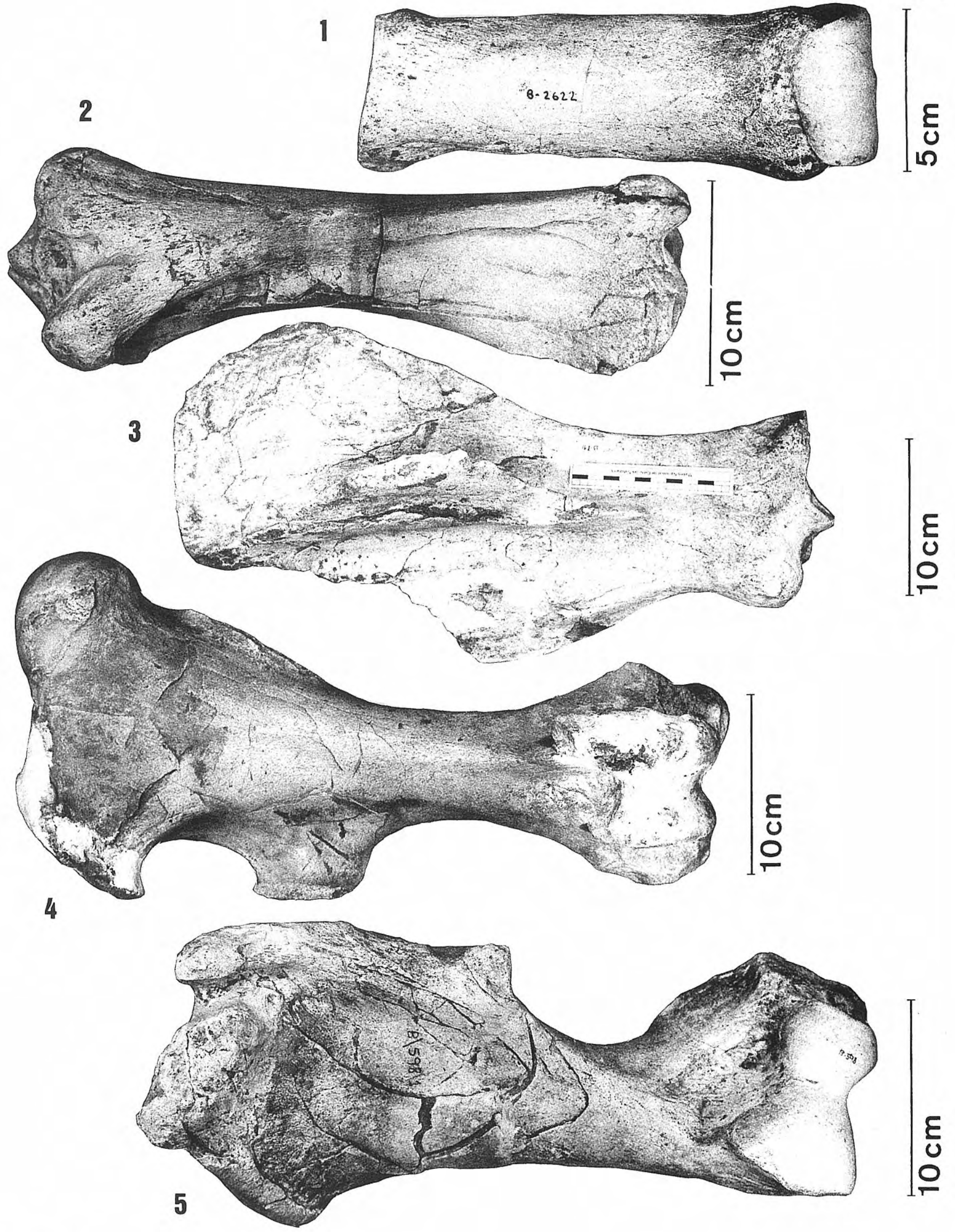




\begin{tabular}{||c|rrrrr||}
\hline \hline Escápula & DTart. & DAPart. & DAPapóf. & DAPmín. & Hmáx. \\
\hline \hline CBAT & 71,0 & 80,0 & 120,3 & 102,2 & 427,0 \\
\hline
\end{tabular}

\begin{tabular}{|c|c|c|c|c|c|c|c|c|c|c|c|}
\hline \multirow[b]{2}{*}{ Húmero } & \multirow[b]{2}{*}{$I$} & \multicolumn{2}{|c|}{ ep.prox. } & \multirow{2}{*}{\multicolumn{2}{|c|}{$\begin{array}{l}\text { art.prox. } \\
\text { DT DAP }\end{array}$}} & \multirow{2}{*}{$\begin{array}{c}\text { cr.d. } \\
\text { DT }\end{array}$} & \multicolumn{2}{|c|}{ diáfisis } & \multirow[b]{2}{*}{ DTm.d. } & \multicolumn{2}{|c|}{ art.dis. } \\
\hline & & & DAP & & & & $\mathrm{DT}$ & DAP & & DT & DAP \\
\hline СВAT & 379,8 & 175 & (101) & 79,0 & 80,5 & 127,6 & 65,8 & 63,0 & 134,7 & 100,0 & 90,8 \\
\hline \multirow[b]{2}{*}{ Fémur. } & \multirow[b]{2}{*}{ I } & \multicolumn{2}{|c|}{ ep.prox. } & \multicolumn{2}{|c|}{ art.prox. } & \multirow{2}{*}{$\begin{array}{c}3 t r . \\
\text { DT. }\end{array}$} & \multicolumn{2}{|c|}{ diáfisis } & & \multicolumn{2}{|c|}{ art.dis. } \\
\hline & & $\mathrm{DT}$ & DAP & DT & DAP & & $\mathrm{DT}$ & DAP & DTm.d & $\mathrm{DT}$ & DAP \\
\hline СВAT & $\begin{array}{l}434,0 \\
425,8\end{array}$ & $\begin{array}{l}176,8 \\
204,0\end{array}$ & $\begin{array}{l}72,0 \\
80,0\end{array}$ & $\begin{array}{l}81,0 \\
87,1\end{array}$ & $\begin{array}{l}69,1 \\
68,0\end{array}$ & $\begin{array}{l}119,0 \\
129,0\end{array}$ & $\begin{array}{l}61,9 \\
64,1\end{array}$ & $\begin{array}{l}50,0 \\
46,6\end{array}$ & $\begin{array}{l}106,9 \\
129,5\end{array}$ & $\begin{array}{r}94,6 \\
110,6\end{array}$ & $\begin{array}{l}74,5 \\
70,5\end{array}$ \\
\hline CLLO & $\begin{array}{l}438,5 \\
445,8 \\
471,0\end{array}$ & $\begin{array}{c}160,0 \\
193,2 \\
-\end{array}$ & $\begin{array}{l}- \\
- \\
-\end{array}$ & $\begin{array}{l}70,0 \\
81,5 \\
82,0\end{array}$ & $\begin{array}{l}65,0 \\
78,0 \\
70,0\end{array}$ & $\begin{array}{l}102,0 \\
119,0 \\
141,0\end{array}$ & $\begin{array}{l}52,0 \\
66,0 \\
66,0\end{array}$ & $\begin{array}{l}44,0 \\
50,0 \\
50,0\end{array}$ & $\begin{array}{l}109,0 \\
121,0 \\
128,0\end{array}$ & $\begin{array}{l}- \\
-\end{array}$ & $\begin{array}{l}- \\
-\end{array}$ \\
\hline
\end{tabular}

\begin{tabular}{||c|ccccccccc||}
\hline \multirow{2}{*}{ Tibia } & \multicolumn{1}{|c|}{ ep.prox. } & \multicolumn{2}{c|}{ diáfisis } & \multicolumn{2}{c|}{ ep.dis. } & \multicolumn{2}{c|}{ art.dis. } \\
& L & DT & DAP & DT & DAP & DT & DAP & DT & DAP \\
\hline \hline \multirow{2}{*}{ CBAT } & 346,6 & 114,8 & 86,7 & 62,7 & 43,4 & 101,0 & 61,8 & 76,5 & 55,7 \\
\hline \multirow{3}{*}{ CLLO } & 370,0 & 121,0 & 108,0 & 52,0 & 49,0 & 91,0 & 67,0 & & \\
& 390,0 & - & 109,0 & 46,0 & 46,0 & - & 64,0 & & \\
& - & 127,0 & 100,0 & - & - & - & - & & \\
\hline
\end{tabular}

\begin{tabular}{||c|ccc||}
\hline \hline Rótula & DT & DAP & H \\
\hline \hline CBAT & 94,5 & 43,7 & 97,4 \\
\hline CLLO & 84,0 & 42,0 & 80,0 \\
& 92,0 & 47,0 & 83,0 \\
\hline
\end{tabular}

Tabla 3. Dimensiones de parte del esqueleto postcraneal de A. incisivum del Cerro de los Batallones. Se incluyen las dimensiones de los ejemplares de Can Llobateres (Santafé, 1978).

la espina escapular se sitúa lejos de la articulación; la espina es triangular y curvada hacia atrás. El tubérculo supraglenoideo es grande y redondeado, bien diferenciado de la apófisis coracoides que sobresale medialmente por encima de la articulación. La faceta articular es grande y bastante redondeada.

El húmero es grande (Tab. 3; Lám. II, fig. 5), con fuertes trocánteres y tuberosidades. La cresta deltoidea es alta, formando un saliente hasta la mitad de la diáfisis. Epicóndilo lateral no muy saliente. Fosa coronoides superficial; fosa olecraniana profunda y ancha.

El fémur izquierdo está algo deformado, pero es posible que pertenezca al mismo individuo que el ejemplar derecho, aunque parece más estrecho (Tab. 3; Lám. II, fig. 4). La epífisis proximal es ancha. El tercer trocánter es bastante saliente y curvado anteriormente. La polea tiene el labio medial muy largo y saliente. La epífisis distal no es muy ancha. La longitud del tercer trocánter, en el ejemplar derecho, es de $65,5 \mathrm{~mm}$.

La tibia es grande, de epífisis no muy anchas (Tab. 3; Lám. II, fig. 2). El surco tibial es ancho. La tuberosidad antero-lateral es robusta. Faceta proximal lateral con poco diámetro antero-posterior. Cresta proximal acusada.

La rótula es ancha y de poco DAP (Tab. 3; Lám. I, fig. 6). Es muy asimétrica y con tuberosidad proximal alta.

El carpo está representado únicamente por el escafoides y el pisiforme (Lám. I, figs. 4-5; Tab. 4). De los dos escafoides, el izquierdo está deformado, pero se aprecia su identidad con el ejemplar derecho, completo y bien conservado. La apófisis anterior forma un acusado ángulo con el cuerpo del hueso. La articulación proximal es larga, poco apuntada lateralmente. Hay tres facetas de articulación con el semilunar; la posterior es larga, aplastada, situada sobre la tuberosidad posterolateral. Las facetas distales son grandes; la del magno se prolonga lateralmente; la del trapezoides es poco convexa; la del trapecio es muy amplia y su borde superior se pierde bajo la tuberosidad postero-lateral.

El pisiforme es robusto, corto, con la articulación ancha (Tab. 4).

Los huesos tarsales (Tab. 4) recuperados son el navicular, el cuboides, el entocuneiforme y el ectocuneiforme; se encontraron formando un conjunto articulado que se ha mantenido como tal, por lo que algunas facetas articulares no se pueden apreciar. 


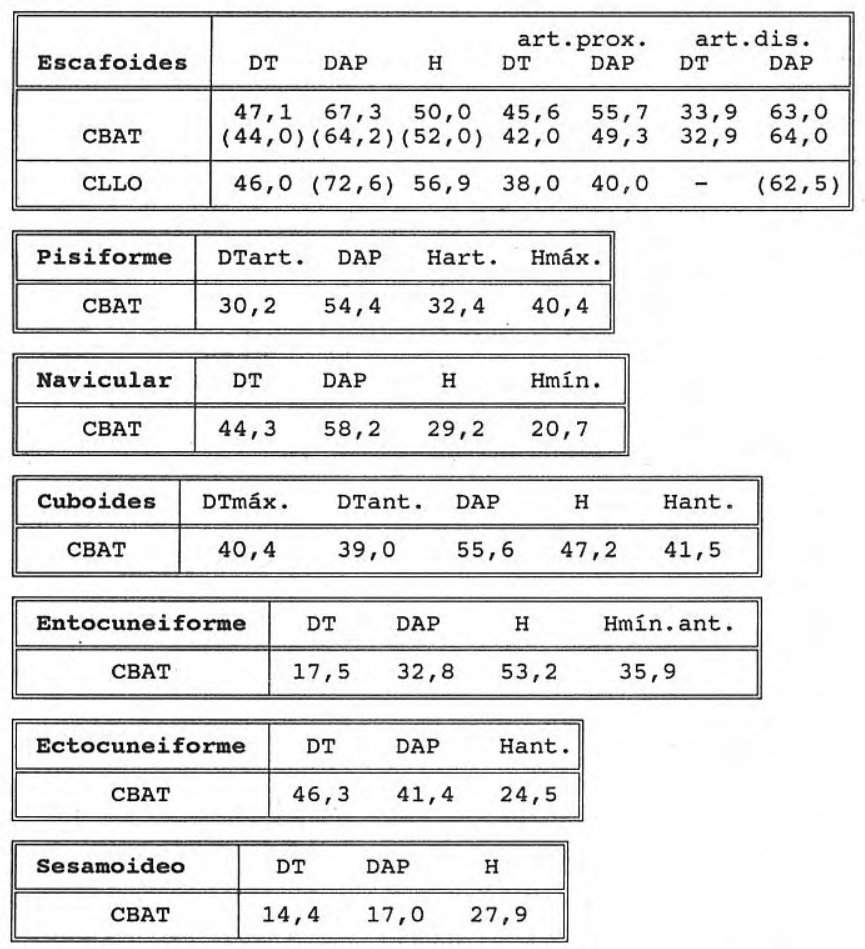

Tabla 4. Dimensiones de los huesos del carpo, del tarso y el sesamoideo de A. incisivum del Cerro de los Batallones. Se incluyen ejemplares de Can Llobateres (Santafé, 1978).

El navicular es romboidal, con la faceta proximal grande, apuntada latero-anteriormente; su borde posterior se inclina muy ligeramente hacia abajo. Las facetas distales tienen crestas muy diferenciadas entre ellas. La inclinación de la faceta para el entocuneiforme es acusada, formando una fuerte cresta con la faceta para el mesocuneiforme.

El cuboides es de cara anterior bastante cuadrada, con el borde superior inclinado medialmente. Es corto anteroposteriormente, con la apófisis posterior poco saliente hacia atrás y distalmente. La faceta para el calcáneo es regularmente convexa hacia el lateral. Un ligero surco limita las dos facetas proximales; no se prolongan apenas en su borde posterior. La faceta distal es corta.

El entocuneiforme es grande; tiene una faceta proximal grande para el navicular (la del mesocuneiforme no se ve). En posición antero-medial se sitúa la faceta para el MtII. El cuerpo del hueso tiene gran diámetro antero-posterior; la apófisis distal es aplastada y corta.

El ectocuneiforme es ancho y corto (DAP), con la cara anterior relativamente alta. La faceta medial superior es pequeña y las del MtII están mal conservadas. Las facetas laterales no se ven.

Metápodos (Tab. 5). El MtII tiene las epífisis estrechas; es relativamente grácil, aunque la diáfisis tiene gran diámetro antero-posterior. La faceta proximal es larga y estrecha. Hay una gran faceta para el entocuneiforme, ovalada. Las facetas laterales, ovaladas, no muestran una subdivisión clara y no se aprecia bien en que proporción articularían con el MtIII y con el ectocuneiforme.

El MtIII (Lám. II, fig. 1) está algo deformado distalmente. La articulación proximal se inclina medialmente; es ancha y corta. Facetas mediales y laterales grandes; falta la faceta lateral posterior y, por tanto, el extremo posterior de la epífisis.

El MtIV tiene la epífisis proximal muy ancha y corta (en DAP), con fuerte torsión respecto a la diáfisis. La tuberosidad lateral es elevada, ensanchando la epífisis y el principio de la diáfisis. Las facetas mediales están muy próximas entre sí, la posterior sobresaliendo hacia atrás. La epífisis distal es estrecha.

Falanges (Tab. 5). La primera falange central tiene un reborde anterior, como prolongación de la faceta articular; el surco medio está marcado sólo en el borde posterior. La primera falange del MtII es estrecha, con DAP grande y una arista anterior en la zona medial. La segunda falange lateral es irregular, más larga en DAP y apuntada lateralmente; es baja.

Las dimensiones del sesamoideo aparecen en la tabla 4.

\section{DISCUSIÓN}

Comparada con los ejemplares tipo de A. incisivum de Eppelsheim, Alemania (Kaup, 1834; moldes estudiados por E.C. en el American Museum of Natural History, New York: AMNH 98036 y AMNH 1000), la mandíbula de Cerro de los Batallones coincide en el perfil inferior rectilíneo, con la sínfisis poco elevada, la rama horizontal larga y relativamente baja, la rama ascendente alta, en ángulo próximo a $90^{\circ}$, el cóndilo ancho, la sínfisis ancha pero poco divergente, con diastemas agudos. Difiere, en cambio, en que la sínfisis es más larga que en los ejemplares tipo, en los cuales alcanza el nivel de $\mathrm{P}_{2}$. En este rasgo difiere asimismo de la descripción que da Guérin (1980) para A. incisivum, lo cual se traduce en una clara diferencia de tamaño y proporción. En general, los ejemplares tipo estudiados son de talla mayor que la mandíbula de Cerro de los Batallones, pero ésta presenta dimensiones que entran en el rango de variación que proporciona Guérin (1980). Sin embargo, la longitud de la sínfisis supera los valores máximos en ambos casos. Por el contrario, la altura de la rama ascendente, tanto en el cóndilo como en la apófisis, presenta valores claramente por debajo de los mínimos señalados por el citado autor. El ejemplar de Can Llobateres descrito por Santafé (1978) presenta también dimensiones mayores que la mandíbula estudiada, salvo en la longitud de la sínfisis que, en este caso, es especialmente corta (Tabla 1), claramente por debajo del mínimo que establece Guérin (op. cit.). La altura de la rama horizontal es menor en Cerro de los Batallones, si bien hay que tener en cuenta la deformación que presenta.

Los dientes yugales de las mandíbulas de Eppelsheim presentan igualmente surco externo marcado pero amplio, sobre todo en los premolares, y no tienen cíngulos laterales. Guérin (1980: p. 242) señala variación en la presencia de estos cíngulos en los premolares de $A$. incisivum. La ausencia de $\mathrm{P}_{1}$ en la mandíbula de Cerro de los Batallones y la presencia de un $\mathrm{P}$, aislado coincide con la variación observada entre los ejemplares de Eppelsheim, ya que la mandíbula AMNH 1000 conserva los $\mathrm{P}_{\text {, }}$ reducidos, mientras que están ausentes en AMNH 98036. 


\begin{tabular}{|c|c|c|c|c|c|c|c|c|c|c|}
\hline \multirow[b]{2}{*}{ MtII } & \multirow[b]{2}{*}{ L } & \multicolumn{2}{|c|}{ ep.prox. } & \multirow{2}{*}{\multicolumn{2}{|c|}{$\begin{array}{l}\text { art.prox. } \\
\text { DT DAP }\end{array}$}} & \multicolumn{2}{|c|}{ diáfisis } & \multirow{2}{*}{$\begin{array}{l}\text { DTmáx. } \\
\text { distal }\end{array}$} & \multicolumn{2}{|c|}{ art.dis. } \\
\hline & & $\mathrm{DT}$ & DAP & & & & & & & DAP \\
\hline CBAT & 128,8 & 25,4 & 40,2 & 18,9 & 34,8 & 20,8 & 26,7 & 27,2 & 27,2 & 38,1 \\
\hline EPPE & 134,6 & - & - & - & - & 25,1 & 21,1 & 37,2 & 34,4 & 37,6 \\
\hline $\begin{array}{l}\text { MtIII } \\
\text { CBAT }\end{array}$ & 152,3 & 49,4 & - & 44,6 & $(38,9)$ & 42,0 & 23,0 & 51,0 & 45,1 & 38,9 \\
\hline EPPE & $\begin{array}{l}157,9 \\
154,1 \\
154,1\end{array}$ & $\begin{array}{l}51,9 \\
48,5 \\
48,4\end{array}$ & $\begin{array}{l}43,9 \\
40,5 \\
41,1\end{array}$ & $\begin{array}{l}48,2 \\
46,6 \\
45,1\end{array}$ & $\begin{array}{l}39,6 \\
37,8 \\
37,2\end{array}$ & $\begin{array}{l}45,7 \\
46,3 \\
46,5\end{array}$ & $\begin{array}{l}20,5 \\
21,1 \\
20,7\end{array}$ & $\begin{array}{l}60,3 \\
62,0 \\
62,2\end{array}$ & $\begin{array}{l}49,0 \\
47,0 \\
47,7\end{array}$ & $\begin{array}{l}41,6 \\
43,0 \\
41,9\end{array}$ \\
\hline CLLO & $\begin{array}{c}165,0 \\
-\end{array}$ & $\begin{array}{l}54,0 \\
42,5\end{array}$ & $\begin{array}{l}45,5 \\
38,5\end{array}$ & & & $\begin{array}{l}43,5 \\
43,5\end{array}$ & $\begin{array}{l}24,0 \\
21,5\end{array}$ & $\begin{array}{l}57,0 \\
49,0\end{array}$ & $\begin{array}{l}45,8 \\
43,0\end{array}$ & $\begin{array}{l}43,0 \\
39,0\end{array}$ \\
\hline $\begin{array}{l}\text { MtIV } \\
\text { CBAT }\end{array}$ & 131,7 & 46,8 & 37,6 & 38,5 & 30,5 & 27,0 & 21,4 & 27,5 & 27,2 & 36,3 \\
\hline CLLO & $\begin{array}{l}137,0 \\
136,0 \\
128,0\end{array}$ & $\begin{array}{c}39,0 \\
37,0 \\
-\end{array}$ & $\begin{array}{l}39,0 \\
40,0 \\
-\end{array}$ & & & $\begin{array}{l}29,0 \\
27,0 \\
26,0\end{array}$ & $\begin{array}{c}22,0 \\
23,0 \\
-\end{array}$ & $\begin{array}{l}\overline{2}, 0 \\
-\end{array}$ & $\begin{array}{l}27,0 \\
28,0 \\
-\end{array}$ & $\begin{array}{l}37,0 \\
37,0 \\
37,5\end{array}$ \\
\hline
\end{tabular}

\begin{tabular}{|c|c|c|c|c|}
\hline $1 a$ & Fal.III & $\mathrm{DT}$ & DAP & $\mathrm{H}$ \\
\hline & CBAT & 47,5 & 34,6 & 37,4 \\
\hline & CBAT & $\begin{array}{l}48,0 \\
45,0 \\
42,0\end{array}$ & $\begin{array}{l}28,3 \\
29,0 \\
31,0\end{array}$ & $\begin{array}{l}36,5 \\
38,0 \\
36,0\end{array}$ \\
\hline $2 a$ & $\begin{array}{l}\text { Fal. III } \\
\text { CBAT }\end{array}$ & 46,0 & 24,2 & 25,0 \\
\hline & CLLO & $\begin{array}{r}41,0 \\
49,0 \\
48,8 \\
\overline{-}, 0\end{array}$ & $\begin{array}{l}23,0 \\
24,0 \\
21,2 \\
22,5 \\
18,2\end{array}$ & $\begin{array}{l}25,0 \\
26,6 \\
24,3 \\
24,5 \\
18,0\end{array}$ \\
\hline
\end{tabular}

\begin{tabular}{||c|ccc||}
\hline \hline 1a Fal.MtII & DT & DAP & H \\
\hline \hline CBAT & 29,2 & 31,4 & 28,3 \\
\hline \hline 2a Fal.MtII & 28,8 & 24,7 & 20,4 \\
\hline CBAT & 39,5 & 26,0 & 22,7 \\
\hline CLLO & 34,5 & 23,5 & 22,0 \\
\hline
\end{tabular}

Tabla 5. Dimensiones de los metápodos y falanges de A. incisivum del Cerro de los Batallones. Se incluyen las dimensiones de los ejemplares de Eppelsheim (moldes del material tipo) y de Can Llobateres (Santafé, 1978).

Las dimensiones tomadas en los dientes (Tab. 2) se incluyen en los rangos de variación que establece Guérin (1980: tab. 50) para las series dentarias de A. incisivum; si se comparan con las dimensiones de dientes aislados (op. cit., tab. 49), la longitud está siempre por debajo de los valores mínimos, salvo en el caso del $\mathrm{P}_{2}$. Los dientes aislados de A. incisivum de diversos yacimientos españoles (Cerdeño, 1989) presentan dimensiones similares a los de Cerro de los Batallones. En cuanto a la longitud relativa de las series premolar $\left(\mathrm{p}_{2}-\mathrm{p}_{4}\right)$ y molar (Tabla 2), la proporción es muy similar en Cerro de los Batallones $(\mathrm{p} / \mathrm{m}=0,78 ; 0,79)$, Can Llobateres $(0,82)$ y Eppelsheim $(0,80 ; 0,79 ; 0,76)$.

Por lo que se refiere al esqueleto postcraneal, tenemos pocos datos comparativos referentes a los huesos largos. Las dimensiones del húmero concuerdan con las del fragmento francés de Soblay, cuya diáfisis es algo más estrecha (Guérin, 1980: tab. 52). El fémur de Höwenegg
(Alemania) señalado por este autor (tab. 65) es más largo y grácil, con el cuello mejor diferenciado, que el de Cerro de los Batallones. Los ejemplares de Can Llobateres son un poco más largos, uno de ellos con la anchura a nivel del tercer trocánter claramente mayor (Tab. 3). La tibia tiene mayor anchura de la diáfisis y de la epífisis distal y menor DAP proximal que los ejemplares de A. incisivum citados por Guérin (1980: tab. 67); los rangos de variación de otros parámetros son muy amplios. Las tibias de Can Llobateres son más grandes en general, pero muestran menor DT de la diáfisis y de la epífisis distal (Tab. 3; Santafé, 1978).

La rótula es relativamente más ancha que la de Soblay (Guérin, 1980: tab. 66) y más alta que las de Can Llobateres (Tab. 3).

El escafoides de Aceratherium (s.l.) carece de la faceta lateral posterior para el semilunar (Santafé, 1978; Guérin, 1980; Cerdeño, 1995: carácter 52), la cual está, 
sin embargo, presente de forma nítida en los ejemplares estudiados. En este carácter, se aproxima al género Lartetotherium, coexistente con A. incisivum en muchos yacimientos europeos del Mioceno superior, aunque la forma y disposición de dicha faceta es diferente. Por otra parte, las dimensiones de nuestros ejemplares son más próximas a A. incisivum que a L. schleiermacheri, de talla claramente mayor y con rasgos morfológicos distintos (Cerdeño, 1989: p. 325). Los ejemplares de Cerro de los Batallones indican, por tanto, cierta variabilidad del carácter comentado dentro del género Aceratherium. Es interesante señalar que esta variación se ha comprobado en una misma población de Acerorhinus zernowi de Tung-gur (China), en la que uno de los tres ejemplares estudiados tiene la tercera faceta bien delimitada y los otros dos la presentan más como una zona de contacto que no llega a ser articulación bien definida; al mismo tiempo, la faceta correspondiente en los semilunares estudiados no existe (Cerdeño, 1995; 1996). La obtención de nuevos restos de Cerro de los Batallones confirmaría si esta morfología peculiar constituye un rasgo individual o si puede generalizarse a nivel de población.

El pisiforme es más ancho, corto y bajo que el ejemplar de Montredon que Guérin (1980: tab. 57) atribuye a $A$. incisivum.

El cuboides es más corto y de cara anterior más alta que las dimensiones que Guérin (1980) establece para $A$. incisivum; en ambos parámetros se aproxima más a los que dicho autor señala para Acerorhinus tetradactylum. Es muy similar en talla y morfología al ejemplar de $A$. cf. incisivum de Los Valles de Fuentidueña (Alberdi et al., 1981; Cerdeño, 1989).

El navicular se ajusta bien a las dimensiones de $A$. incisivum (Guérin, 1980; Cerdeño, 1989), con una longitud (DAP) relativamente algo mayor que en los ejemplares de Concud y el Vallés-Penedés (Cerdeño, 1989: tab. 31).

El entocuneiforme tiene una talla comparable al único ejemplar descrito de A. incisivum, procedente de La Roma (Cerdeño, 1989; Cerdeño y Alcalá, 1989). El ectocuneiforme, en cambio, es más pequeño que el de este yacimiento y algo más ancho que los publicados por Guérin (1980).

Los metápodos son comparables a los de A. incisivum descritos en otros yacimientos españoles (Santafé, 1978; Cerdeño, 1989), así como a los moldes estudiados de Eppelsheim (MtII, AMNH 98025; MtIII, AMNH 98027, 98041 y 98056). El MtII de Cerro de los Batallones tiene la faceta proximal un poco más larga relativamente y la longitud total es menor que en el ejemplar alemán, aunque entra en la variación de los dos MtII del VallésPenedés. El MtIII es similar a los de Eppelsheim, pero con menores diámetros distales. El MtIV tiene mayor anchura proximal por el desarrollo de la tuberosidad lateral. Los tres metatarsos tienen la epífisis distal relativamente estrecha. Respecto a los datos que proporciona Guérin (1980), las dimensiones del MtII rondan los valores medios, salvo los diámetros transversales distales que son claramente inferiores, por debajo de los mínimos. Con el MtIV ocurre algo similar, teniendo además mayor DT proximal. El MtIII tiene menos diferencia en las dimensiones distales, entrando en el rango de variación que da el citado autor; la diáfisis tiene un DAP relativamente mayor. Los metápodos de Can Llobateres son más largos, especialmente el MtIII (Tab. 5). Respecto a las diferencias observables en los diámetros de la diáfisis del MtII (Tabla 5), no son tales sino que se deben al criterio de medida (ver Metodología). El índice de gracilidad (100 x DT diáfisis/ L) calculado para estos metápodos $(\mathrm{MtII}=16,14 ; \mathrm{MtIII}=$ 27,57; MtIV = 20,5) es comparable a los obtenidos en otros ejemplares de A. incisivum españoles (Cerdeño, 1989: 160) y de Eppelsheim (MtII: 15,67; MtIII: 28,9; $30,0 ; 30,1)$.

\section{CONCLUSIONES}

Tras el estudio comparativo detallado de los restos de rinoceronte del yacimiento de Cerro de los Batallones, se confirma su atribución a la especie Aceratherium incisivum. Presentan una talla menor que el material tipo de Eppelsheim, pero entran en la variación conocida para la especie. Destaca la mayor longitud de la sínfisis mandibular, que supera los máximos establecidos, al tiempo que la altura de la rama ascendente es relativamente más baja que en otros ejemplares. Hay que destacar también la morfología del escafoides, debido a que presenta una faceta latero-posterior bastante bien definida, carácter que no es propio de Aceratherium, aunque se conoce algún caso en el género Acerorhinus, muy próximo al anterior.

\section{AGRADECIMIENTOS}

A los Drs. S. Roig y L. Alcalá por la lectura crítica del manuscrito. Los moldes del material de Eppelsheim incluidos en este trabajo pertenecen a la colección de vertebrados del AMNH (New York), a cargo del Dr. R. H. Tedford. Al laboratorio de Restauración del Museo Nacional de Ciencias Naturales, por la labor realizada sobre el material estudiado. Agradecemos la colaboración de L. de Luque (Dpto. Paleobiología) y el laboratorio de Fotografía, del M.N.C.N. en la elaboración de parte del material fotográfico. Las excavaciones del Cerro de los Batallones han sido financiadas por la Consejería de Educación y Cultura de la Comunidad Autónoma de Madrid. Este trabajo se ha realizado dentro del Proyecto PB-94 0071 de la DGICYT.

\section{BIBLIOGRAFÍA}

Alberdi, M.T., Ginsburg, L. y Morales, J. 1981. Rhinocerotidae del yacimiento de los Valles de Fuentidueña (Segovia). Estudios geológicos, 37, 439465.

Borissiak, A. 1927. Aceratherium depereti n. sp. from the Jilancik beds. Bulletin of the Academy of Sciences URSS, 21(6), 769-786. 
Cerdeño, E. 1989. Revisión de la Sistemática de los rinocerontes del Neógeno de España. Colección Tesis Doctorales, $\mathrm{N}^{\mathrm{O}} \mathbf{3 0 6 / 8 9}$. Edit. Universidad Complutense de Madrid, 429 pp.

Cerdeño, E. 1992. Spanish Neogene rhinoceroses. Palaeontology, 35, 297-308.

Cerdeño, E. 1993. Rinocerontes fósiles en la Comunidad de Madrid. In: Madrid antes del Hombre. Eds. Museo Nacional de Ciencias Naturales, CSIC, y Comunidad de Madrid, 46-48.

Cerdeño, E. 1995. Cladistic analysis of the Family Rhinocerotidae. American Museum Novitates, 3143, 25 pp.

Cerdeño E. 1996. Rhinocerotidae from the Middle Miocene of the Tung-gur Formation, Inner Mongolia (China). American Museum Novitates, 3184, 43 pp.

Cerdeño, E. y Alcalá, L. 1989. Aceratherium alfambrense sp. n., nuevo rinocerótido del Vallesiense superior de Teruel (España). Revista Española de Paleontología, 4, 39-51.

Ginsburg, L. and Heissig, K. 1989. Hoploaceratherium n. gen., a new generic name for "Aceratherium" tetradactylum. In: The evolution of Perissodactyls. (Eds. D. R. Prothero and R. M. Schoch). New York, Oxford University Press, 418-421.

Guérin, C. 1980. Les rhinocéros (Mammalia, Perissodactyla) du Miocène terminal au Pléistocène supérieur en Europe occidentale. Comparaison avec les espèces actuelles. Documents du Laboratoire de Géologie de Lyon, 79 (13), $1182 \mathrm{pp}$.

Kaup, J.J. 1834. Description d'ossements fossiles de mammifères inconnus jusqu'à présent qui se trouvent au
Museum Gran Ducal de Darmstadt. $3^{e}$ cahier: Rhinoceros schleiermacheri, Acerorhinus incisivum, Aceratherium goldfussi. J. G. Heyer, édit., 33-64.

Kretzoi, M. 1942. Bemerkungen zum System der nachmiozänen Nashorn-Gattungen. Földtani Közlöny, 72, 309-318.

Lartet, E. 1851. Notice sur la colline de Sansan. J. A. Portes édit., Auch, 45 pp.

Morales, J., Capitán, J., Calvo J.P. y Sesé, C. 1992. Nuevo yacimiento de vertebrados del Mioceno Superior al sur de Madrid (Cerro Batallones, Torrejón de Velasco). Geogaceta, 12, 77-80.

Morales, J., Alcalá, L. y Nieto, M. 1993. Las faunas de vertebrados del Terciario. In: Madrid antes del Hombre. Eds. Museo Nacional de Ciencias Naturales, CSIC, y Comunidad de Madrid, 23-31.

Santafé, J.V. 1978. Rhinocerótidos fósiles de España. Tesis doctoral inédita. Universidad Central de Barcelona, 489 pp.

Santafé, J.V. y Casanovas, M.L. 1982. Los rinocerótidos (Mammalia, Perissodactyla) del Turoliense del Penedés (Piera, Barcelona). Bolletín Informativo Instituto de Paleontología de Sabadell, 14 (1-2), 39-47.

Santafé, J.V. y Casanovas, M.L. 1984. Elementos del esqueleto postcraneal de Dicerorhinus schleiermacheri y Aceratherium incisivum (Perissodactyla, Rhinocerotidae) de la localidad turoliense de Concud (Teruel). Paleontologia i Evolució, 18, 95-103.

Santafé, J.V. y Casanovas, M.L. 1992. Los rinocerótidos (Mammalia, Perissodactyla) de la localidad vallesiense de Polinyá (Barcelona). Treballs Museu Geologia de Barcelona, 2, 45-67. 